\title{
Measuring eccentricity in binary black-hole initial data
}

\author{
Jason D. Grigsby and Gregory B. Cook \\ Department of Physics, Wake Forest University, Winston-Salem, North Carolina 27109
}

(Dated: November 11, 2018)

\begin{abstract}
Initial data for evolving black-hole binaries can be constructed via many techniques, and can represent a wide range of physical scenarios. However, because of the way that different schemes parameterize the physical aspects of a configuration, it is not alway clear what a given set of initial data actually represents. This is especially important for quasiequilibrium data constructed using the conformal thin-sandwich approach. Most initial-data studies have focused on identifying data sets that represent binaries in quasi-circular orbits. In this paper, we consider initial-data sets representing equal-mass black holes binaries in eccentric orbits. We will show that effective-potential techniques can be used to calibrate initial data for black-hole binaries in eccentric orbits. We will also examine several different approaches, including post-Newtonian diagnostics, for measuring the eccentricity of an orbit. Finally, we propose the use of the "Komar-mass difference" as a useful, invariant means of parameterizing the eccentricity of relativistic orbits.
\end{abstract}

PACS numbers: 04.20.-q, 04.25.Dm, 04.70.Bw, 97.80.-d

\section{INTRODUCTION}

The possible detection of gravitational waves by detectors such as LIGO and LISA is driving rapid progress in the binary black hole $(\mathrm{BBH})$ problem. The final stages of the inspiral and coalescence are believed to be primary sources of gravitational waves at frequencies accessible by such detectors. Theoretical models that accurately predict these final stages of inspiral are needed to help analyze and improve the rate of detection with future data. There are two techniques commonly used to study such systems, the post Newtonian approximation and numerical relativity. Since post Newtonian methods are expected to break down at smaller separations, accurate models of final stage inspirals need to be handled with numerical techniques.

Numerical relativity breaks the problem into two parts, the specification of initial data and the numerical time evolutions of this data. With recent advances in evolutions 1, 2, 3, 4], it is as important as ever to fully understand the initial data one is starting with. Post Newtonian methods have shown that BBHs starting with large separation will evolve toward an adiabatic inspiral that follows a series of quasi-circular orbits [5]. This quasicircular inspiral is thought to last till late stages where the black holes plunge toward coalescence. Most work to date in both the construction of $\mathrm{BBH}$ initial data and evolutions have focused on quasi-circular configurations. But the study of binaries in truly eccentric orbits near coalescence may be important for gravity-wave detectors (especially LISA) and, in any case, is of considerable theoretical interest. A primary goal of this paper is to take a preliminary look at how current quasiequilibrium methods for constructing BBH initial data can be extended to

\footnotetext{
*Electronic address: grigjd3@wfu.edu
}

${ }^{\dagger}$ Electronic address: cookgb@wfu.edu construct general eccentric configurations.

To predict quasicircular orbits, two techniques have commonly been used: the effective-potential (EP) method [6] and the Komar-mass ansatz [7]. The Komarmass ansatz compares two definitions of energy: the ADM energy [8] and the Komar mass [9]. The ADM energy gives a proper definition of energy at spatial infinity in all cases, but the Komar mass is only accurate when a configuration is at least momentarily stationary. So, the Komar-mass ansatz posits that when these two energies are equal, the system should be in quasiequilibrium. The EP technique is motivated by the Newtonian effective one-body problem, and determines a quasi-circular configuration by finding models that have a minimum in the binding energy along a sequence of constant angular momentum, constant masses, and constant spins. The two methods have been contrasted and largely agree, with significant differences occurring only at separations close to final plunge of the BBHs 10, 11].

It has been conjectured 10] that the EP method, when used with methods for constructing quasiequilibrium initial data, should generalize to represent BBHs in eccentric orbits. For initial-data methods designed to produce quasiequilibrium data, the generalization is not obvious because the individual black holes will not follow the integral curves of any approximate Killing field. We will explore this conjecture extensively in this paper. In order for this conjecture to be true, the initial data used to construct the effective potentials must represent BBHs at turning points in their orbits. Unfortunately, we cannot test this property of the data directly. We will justify the assumption that the data represent BBHs at turning points and test it by comparison to various results from post-Newtonian methods.

By constructing effective-potentials that represent $\mathrm{BBHs}$ in eccentric orbits, it becomes possible to make direct estimates of the eccentricity of an orbital configuration. Of course, it is not possible to justify a unique definition for eccentricity for relativistic configurations 
where elliptic orbits do not exist. Instead, we compare several different definitions [12] and show that they give reasonable and consistent results. Because there is no unique definition of eccentricity, it is perhaps not the best quantity for parameterizing eccentric orbits. We find that the "Komar-mass difference", the quantity that is set to zero in the Komar-mass ansatz for defining circular orbits, may serve as a useful parameterization of eccentric orbits.

We begin in Sec. III with an overview of the quasiequilibrium method we use to construct $\mathrm{BBH}$ initial data, emphasizing the aspects that will be most relevant to our subsequent discussion. In Sec. III, we will discuss various aspects of effective potentials in the context of their use with $\mathrm{BBH}$ initial data. In particular, we will justify as fully as we can the extension of these effective potentials to BBHs in eccentric orbits. In Sec. IV we will explore and compare several definitions of eccentricity, and will motivate the use of the "Komar-mass difference" as an invariant means of parameterizing BBHs in eccentric orbits. All of the preceding discussions have dealt with non-spinning black holes. In Sec. V] we will briefly revisit the case of corotating black holes. We end the paper in Sec. VI] with some conclusions.

\section{INITIAL DATA}

The binary black-hole initial-data sets that are used below were described in detail in Refs. [10, 13] and references within. Here, we give an overview of the methods used to construct the initial data, elaborating only on the details most relevant to finding black-hole binaries in quasi-circular orbits.

Our initial-data sets are constructed within the extended conformal thin-sandwich approach [14, 15]. This approach is based on the standard $3+1$ decomposition where the space-time interval is written as

$$
\mathrm{d} s^{2}=-\alpha^{2} \mathrm{~d} t^{2}+\gamma_{i j}\left(\mathrm{~d} x^{i}+\beta^{i} \mathrm{~d} t\right)\left(\mathrm{d} x^{j}+\beta^{j} \mathrm{~d} t\right) .
$$

Here, $\gamma_{i j}$ is the spatial metric, and $\alpha$ and $\beta^{i}$ are the lapse function and shift vector. Minimal initial data for a Cauchy evolution requires that we fully specify $\gamma_{i j}$ and the extrinsic curvature $K_{i j}$ (essentially a first time derivative of the spatial metric) defined as

$$
K_{i j} \equiv-\frac{1}{2} \mathcal{L}_{n} \gamma_{i j},
$$

where $n^{\mu}$ is the time-like unit normal to the $t=$ const. initial-data surface.

The conformal thin-sandwich (CTS) approach requires a conformal decomposition of $\gamma_{i j}$, and that we specify the conformally related metric $\tilde{\gamma}_{i j}$. In this work we will always take $\tilde{\gamma}_{i j}$ to be flat. We must also specify the time derivative of the conformal metric $\partial_{t} \tilde{\gamma}_{i j}$ and the trace of the extrinsic curvature. We fix both quantities to be zero. The CTS approach then requires that we determine the conformal factor $\psi$ relating $\gamma_{i j}$ and the conformal metric, and the shift vector $\beta^{i}$. These are obtained by solving elliptic versions of the Hamiltonian and momentum constraint equations (see Ref. [10] for details). Finally, the extended CTS approach also requires that we determine the lapse function $\alpha$ by fixing the time derivative of the trace of the extrinsic curvature and then solving the evolution equation for the trace of the extrinsic curvature as an elliptic equation. We fix the trace of the extrinsic curvature to be constant in time.

In constructing black-hole initial data, we excise the black-hole interior from the computational domain and must impose boundary conditions at the excision surfaces when solving the elliptic equations for $\psi, \beta^{i}$, and $\alpha$. We demand that each black hole be in quasiequilibrium by imposing the boundary conditions worked out in Refs. [16] and [13]. The assumptions of quasiequilibrium are essentially the same as those required of an "isolated horizon" (see Refs. 17, 18, 19]). We must also impose boundary conditions at the outer boundary of the computational domain (either at infinity or some large radial distance from the black holes). For this, we assume that our configuration is asymptotically flat. However, asymptotic flatness does not fully fix the boundary conditions on the constrained data. The asymptotic condition on the shift is

$$
\left.\beta^{i}\right|_{r \rightarrow \infty}=\left(\boldsymbol{\Omega}_{0} \times \mathbf{r}\right)^{i}
$$

where $\boldsymbol{\Omega}_{0}$ is an angular velocity vector.

The time coordinate threading through our initial-data slice is defined by

$$
t^{\mu} \equiv \alpha n^{\mu}+\beta^{\mu}
$$

Imposing Eq. (3), we see that $\boldsymbol{\Omega}_{0}$ determines the rotation of the "helical" time coordinate. For a binary system in equilibrium, the time coordinate generates a symmetry and the bodies move in circular orbits along integral lines of the time coordinate. For relativistic systems, the binary can only be in quasiequilibrium and the time coordinate generates an approximate symmetry. Nevertheless, $\boldsymbol{\Omega}_{0}$ represents the orbital angular velocity of the binary as measured by observers at infinity. From a computational perspective, $\boldsymbol{\Omega}_{0}$ must be chosen. Ultimately, it is the effect of different choices for $\boldsymbol{\Omega}_{0}$ that we will be exploring in this paper.

In Ref. [10], two independent methods for choosing the magnitude of $\boldsymbol{\Omega}_{0}$ were compared. Both methods attempt to produce a binary system that is in quasiequilibrium with the black holes in quasi-circular orbits. One method is based on the Komar-mass ansatz, first proposed by Gourgoulhon et. al. [7], which posits that if $\Omega_{0}$ is chosen so that the ADM energy $E_{\mathrm{ADM}}$ and the Komar mass $M_{\mathrm{K}}$ of a system are equal, then the system will be nearly stationary (i.e. in quasiequilibrium) and the binary will 
be in a quasi-circular orbit. These masses are defined via

$$
\begin{aligned}
E_{\mathrm{ADM}} & =\frac{1}{16 \pi} \oint_{\infty} \nabla_{j}\left(\mathcal{G}_{i}^{j}-\delta_{i}^{j} \mathcal{G}\right) d^{2} S^{i}, \\
M_{\mathrm{K}} & =\frac{1}{4 \pi} \oint_{\infty}\left(\nabla_{i} \alpha-\beta^{j} K_{i j}\right) d^{2} S^{i},
\end{aligned}
$$

where $\mathcal{G}_{i j} \equiv \gamma_{i j}-f_{i j}, f_{i j}$ is the flat metric, and $\nabla_{j}$ is the covariant derivative compatible with $\gamma_{i j}$. The second method assumes that quasi-circular orbits are found at the minima of a reduced two-body effective potential. Effective potentials will be described in more detail in Sec. III.

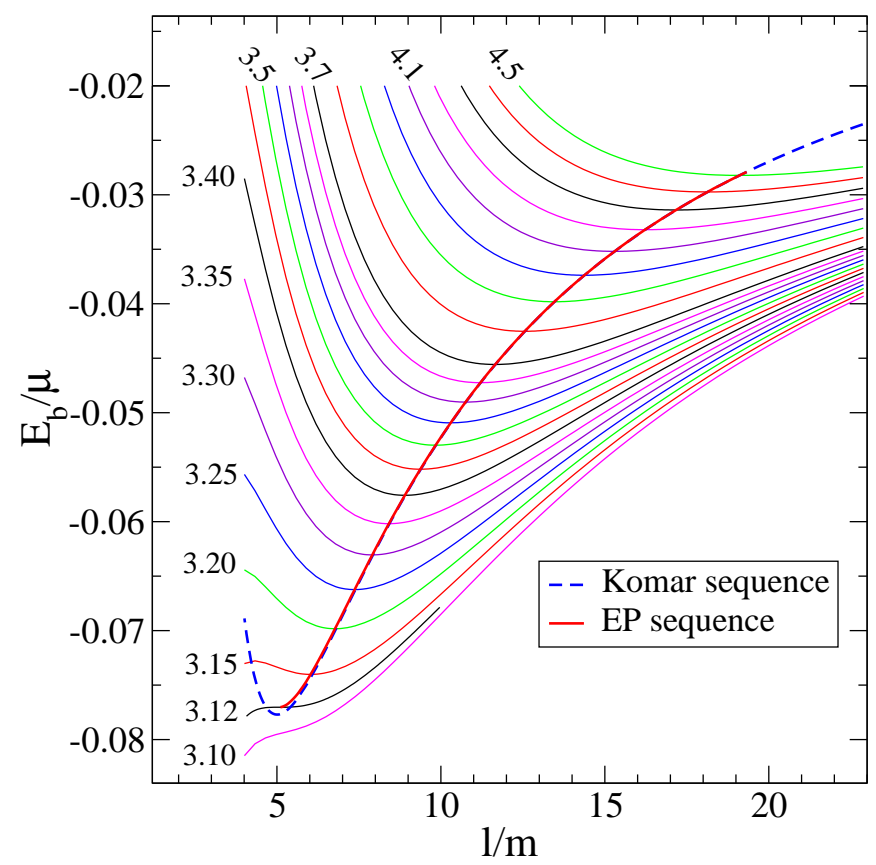

FIG. 1: Effective-potential plot for non-spinning equal-mass black holes constructed from numerical initial data [10]. The thin solid(multi-color) lines, are individual EP curves. Some of these EP curves are labeled it value of $J / \mu m$. Passing through the local minima of these EP curves and drawn as a bold(red) line is the EP sequence of quasi-circular orbits. The Komar sequence of quasi-circular orbits is displayed as a dashed(blue) line.

In Ref. [10], we showed that the circular-orbit configurations produced by both methods agree remarkably well for both non-spinning and corotating black-hole binaries. Here, we simply show a figure that directly compares sets of circular-orbit models as we vary the binary separation. Figure 1 displays the effective potential (EP) curves for non-spinning equal-mass black-hole binaries as computed in Ref. [10]. The vertical axes display the dimensionless binding energy of the binary $E_{\mathrm{b}} / \mu$, where $E_{\mathrm{b}}$ is defined in Eq. (8), $\mu \equiv m_{1} m_{2} / m$ is the reduced mass of the system and $m \equiv m 1+m 2$ is the total mass of the binary. The horizontal axes display the dimensionless proper separation of the binary $\ell / m$. It is important to note that each EP curve consists of a sequence of models where the value of $\Omega_{0}$ changes monotonically. Passing through the minima of the EP curves is the "EP sequence" defined as the sequence of quasi-circular orbit models where quasi-circular orbits are defined via the effective-potential method. Also shown in Fig. 1 is the "Komar sequence" defined as the sequence of quasicircular orbit models defined via the Komar-mass ansatz. It is clear that the two sequences agree quite well except for the smallest separations. See Ref. [10] for a more detailed comparison. Most important for our considerations is that both the Komar-mass and EP methods choose particular models on each EP curve, with very similar values of $\Omega_{0}$, as quasi-circular orbit models.

These sequences of quasi-circular orbit models represent a good approximation to the adiabatic inspiral of a black-hole binary so long as the binary separation is not too small. For large enough separation, the time scale for radiation reaction to induce a significant change in the orbital radius is much larger than the orbital period. At small enough separations, radiation reaction becomes significant and the sequences of quasi-circular obits will eventually become a poor approximation to an adiabatic inspiral. It is difficult to estimate exactly where this transition occurs, but it will certainly occur at separations larger than the inner-most stable circular orbit (ISCO). For a Komar sequence, the ISCO is defined to occur at the minimum in the binding energy. For an EP sequence, the ISCO is defined to occur at the point where the EP curves no longer have a local minimum. Both ISCOs occur near $\ell / m \sim 5$ (see Fig. (1).

When the CTS equations and excision boundary conditions as described above are used to construct initial data for binaries in quasi-circular orbit, the resulting data are consistent with a system in quasiequilibrium. In particular, we have both bodies in the binary following the integral curves of an approximate helical Killing vector (the time coordinate). Furthermore, half of the initial data that must be specified is also consistent with this notion of quasiequilibrium. Recall that the conformal metric $\tilde{\gamma}_{i j}$, the trace of the extrinsic curvature, and their time derivatives must be specified, and that we take all of the time derivatives to vanish. As long as the binary is in a circular orbit, the notion of quasiequilibrium is satisfied.

However, we are left to wonder, what happens if we choose $\Omega_{0}$ so that the binary is not in a quasi-circular orbit? In Ref. [10], we made the assertion that the resulting initial data would represent a binary at either pericenter or apocenter of a general bound or unbound orbit. To arrive at this conclusion, we are forced to give up the notion that the helical time vector represents an approximate Killing vector of the space-time. Our goal below is to see if this interpretation is reasonable. 


\section{EFFECTIVE POTENTIALS}

In Newtonian physics, 1-D effective potentials nicely capture the important features of certain dynamical systems. In the case of the reduced gravitational 2-body problem, for a given orbital angular momentum, the effective potential can be used to locate the turning points for an elliptic orbit of given energy, or the radius and energy of a circular orbit. No such 1-D effective potential can be rigorously and uniquely derived for the fully relativistic gravitational 2-body problem. However, a useful effective potential has been defined [6, 11, 20, 21] in direct analogy with the Newtonian gravitational effective potential.

In essence, an EP curve is the total energy of the system for a sequence of configurations where the radial separation varies, but all other physical parameters are held fixed. In order to correspond to an effective potential, the velocity of the generalized coordinate that is allowed to vary (the radial separation in this case) must vanish so that there is no associated kinetic energy. Binary systems in bound orbits with vanishing radial velocity are either at apocenter or pericenter, and collectively we refer to these as turning points in the orbit.

For black-hole binaries, we use the following functional definition for an EP curve. We consider a sequence of initial-data configurations that hold constant the apparent-horizon masses of the individual black holes $\left(m_{1}\right.$ and $\left.m_{2}\right)$, the magnitude and direction of the spins of the individual black holes, and the total angular momentum of the system ${ }^{1}$. We also require that the binary be at a turning point in the orbit. For the value of the effective potential, we could simply use the total ADM energy of the system. However, it is more intuitive to use the binding energy since this allows us to estimate if a given configuration is in a bound orbit. The binding energy requires that we subtract from the total energy, the total mass of the system at infinite separation (i.e. the sum of the masses of the individual black holes). Unfortunately, there is no rigorous way of defining the total mass of an individual black hole at finite separation. This is a fundamental uncertainty in defining a relativistic effective potential.

The total mass of an individual black hole includes both its irreducible mass and the kinetic energy associated with the spin. In general, we cannot rigorously define the spin of an individual black hole, nor do we know how to compute the total mass of an individual black hole given the spin and apparent-horizon mass. A reasonable approximation is to define the total mass of an individual

\footnotetext{
1 It would be preferable to hold the total mass of the individual black holes constant. However, as we will discuss in the main text, this is not possible.
}

black hole, $M$, via the Christodoulou formula 22$]$

$$
M^{2}=M_{\mathrm{irr}}^{2}+\frac{S^{2}}{4 M_{\mathrm{irr}}^{2}}
$$

where $S$ is the magnitude of the spin of the black hole and we can approximate the irreducible mass, $M_{\mathrm{irr}}$, with the apparent-horizon mass.

To avoid some of these ambiguities, we will define the binding energy as the ADM energy minus the sum of the apparent horizon masses.

$$
E_{b} \equiv E_{\mathrm{ADM}}-m_{1}-m_{2}
$$

Because we keep the apparent-horizon masses constant along an EP curve, this constant shift does not affect the effective potential. But, since we do not keep the total mass constant along an EP curve, the uncertainty in the definition of the total mass contributes to the uncertainty in the definition of an EP curve.

Another fundamental uncertainty in our numerical EP curves is the assumption that they are constructed of turning points in eccentric orbits. When the EP method was introduced, it was applied while using the conformal imaging decomposition which allowed one to directly set the radial momentum of the two holes to zero [6]. In the CTS decomposition, there is no mechanism to directly set such data. However, as discussed at the end of Sec. III, we conjecture that our initial data does have zero radial momentum. The reasoning behind this conjecture is that the time derivative of the conformal metric is still taken to vanish, so that at least instantaneously we expect each black hole to be at a turning point.

In order to test the validity of this conjecture, we compare our numerical effective potentials to post Newtonian data. Mora and Will [12] introduced third order conservative post Newtonian equations for the energy and angular momentum of a system in terms of an eccentricity $\epsilon$ and inverse semilatus rectum $\zeta$. The authors treated the black holes as having zero spin and ignored dissipative terms. One can find the orbital angular velocity at either pericenter or apocenter through the author's choice of definition of $\epsilon$ and $\zeta$ used in creating these equations. In order to include the spin of the black holes, it becomes necessary to include correction terms that create equations of the following form.

$$
\begin{aligned}
E(\epsilon, \zeta, \omega)= & E_{\mathrm{ADM}}(\epsilon, \zeta)+E_{\mathrm{self}}(\epsilon, \zeta, \omega) \\
& +E_{\mathrm{N}, \text { Corr }}(\epsilon, \zeta, \omega)+E_{\mathrm{Spin}}(\epsilon, \zeta, \omega) \\
J(\epsilon, \zeta, \omega)= & J_{\mathrm{ADM}}(\epsilon, \zeta)+S(\epsilon, \zeta, \omega) \\
& +J_{\mathrm{N}, \text { Corr }}(\epsilon, \zeta, \omega)+J_{\mathrm{Spin}}(\epsilon, \zeta, \omega)
\end{aligned}
$$

In these equations $\omega$ represents the spin angular velocities of the black holes ${ }^{2}$. The self energy and spin terms

\footnotetext{
${ }^{2}$ We use a single parameter $\omega$ for simplicity. In general, specifying the spin of two black holes would require six parameters.
} 


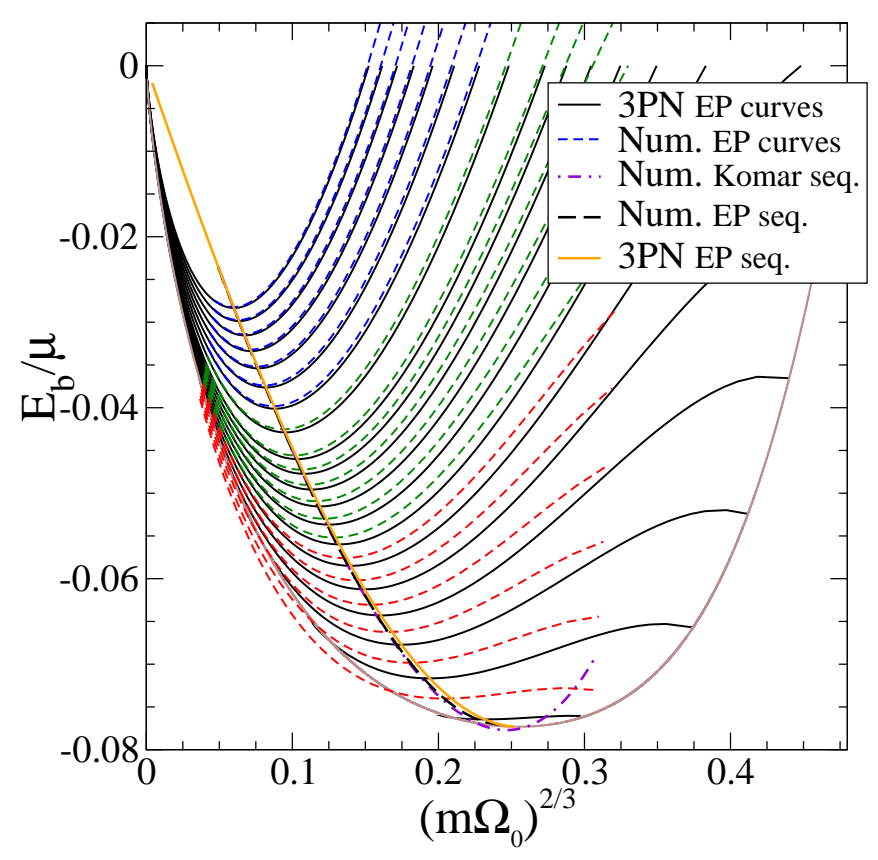

FIG. 2: EP curves for non-spinning equal-mass black holes from both numerical and 3PN data. Numerical EP curves are plotted as short-dashed(blue, green, and red) lines. 3PN EP curves are plotted as solid(black) lines. The Komar sequence through the numerical data is plotted as a dash-dot(purple) line. The EP sequence through the numerical data is plotted as a long-dash(black) line. The EP sequence through the 3PN data is plotted as a light-solid(orange) line. A boundary of the allowable region for the $3 \mathrm{PN}$ equations is shown as a light-solid(brown) line.

$\left(E_{\text {self }}\right.$ and $\left.S\right)$ are derived as expansions of the Kerr formulae relating mass, spin, and rotational angular velocity. The Newtonian correction terms $\left(E_{\mathrm{N}, \text { Corr }}\right.$ and $\left.J_{\mathrm{N}, \text { Corr }}\right)$ stem from the conversion of total mass to irreducible mass and the "Spin" terms $\left(E_{\mathrm{Spin}}\right.$ and $\left.J_{\mathrm{Spin}}\right)$ represent spin-orbit effects. The parameter space of these equations are shown below in Figs. 5 and 6 ,

With this parametrization, one can easily construct 3PN EP curves using straight-forward one-dimensional root finding. To create a sequence of constant angular momentum, one can apply a sequence of values for the eccentricity to Eq. (10), and for each find the corresponding $\zeta$ that returns the desired value of angular momentum. One can then use those values of $\epsilon$ and $\zeta$ in Eq. (9) to find the energy of the system. In Fig. 2, we plot for comparison the EP curves for non-spinning, equal-mass binaries from both the numerical data and the $3 \mathrm{PN}$ equations. The energy is plotted as a function of the dimensionless orbital angular velocity $m \Omega$, where small $m \Omega$ corresponds to large orbital separation. Included on the graph are data from the Komar sequence and the EP sequences extracted from the minima of the $3 \mathrm{PN}$ EP curves and the numerical EP curves.

At large values of angular momentum (large orbital separation), the solid 3PN data agrees well with the dashed numerical data. The numerical data and the 3PN data begin to diverge as the angular momentum decreases. This is not surprising as it is well known the $\mathrm{PN}$ expansion is less accurate for tighter binary systems and the same is true for the numerical initial data models. It seems from the good agreement between numerical and 3PN EP curves that the numerical data we construct using the CTS approach are reasonably close to turning points, and seem to asymptote to turning points as the system becomes more Newtonian.

\section{MEASURING ECCENTRICITY}

The comparison of our numerical EP curves with 3PN EP curves in Sec. III gives us confidence that the numerical data represent systems at either pericenter or apocenter. Since each numerical EP curve is constructed from a sequence of models where the mass, angular momentum and spins are held constant and are at turning points, it is reasonable to use these curves to try to define the eccentricity for a given model. For Newtonian binaries, eccentricity can be measured using relative separation at pericenter $d_{p}$ and apocenter $d_{a}$ :

$$
\epsilon_{d} \equiv \frac{d_{a}-d_{p}}{d_{a}+d_{p}}
$$

We use $\epsilon_{d}$ to denote the eccentricity of numerical models based purely on separations.

By applying Newtonian equations of motion, one can replace the above definition of eccentricity dependent on relative separation with a version that is dependent on the orbital angular velocities at pericenter $\Omega_{p}$ and apocenter $\Omega_{a}$. Not only is the eccentricity found in those terms, but the dimensionless inverse semilatus rectum can also be found using the same parameters [12].

$$
\begin{aligned}
\epsilon_{\Omega} & \equiv \frac{\Omega_{p}-\Omega_{a}}{\Omega_{p}+\Omega_{a}} \\
\zeta & \equiv\left(\frac{\sqrt{m \Omega_{p}}+\sqrt{m \Omega_{a}}}{2}\right)^{4 / 3} .
\end{aligned}
$$

We use $\epsilon_{\Omega}$ to denote the eccentricity of our numerical models as measured using orbital angular velocities. The following relationships follow directly from these equations:

$$
\zeta=\left(\frac{m \Omega_{p}}{(1+\epsilon)^{2}}\right)^{2 / 3}=\left(\frac{m \Omega_{a}}{(1-\epsilon)^{2}}\right)^{2 / 3} .
$$

We note that these equations (at either pericenter or apocenter) are the only places in any of our work where the sign of the eccentricity matters and so for the remainder of the paper we will ignore the middle (pericenter) relationship and assume eccentricity is negative at pericenter. This has the added benefit of cleaning up many of the figures below. 


\section{A. Direct Measurement}

Our next goal is to determine the eccentricities of the data in our EP curves and determine if the definition of eccentricity in Eq. (12) yields reasonable results. We start by computing $\epsilon_{\Omega}$ for the non-spinning equal-mass models represented in the numerical EP curves displayed in Fig. 2 (also seen in Fig. 1). The measured eccentricities are shown in Fig. 3, plotted against $m \Omega$. The $\epsilon_{\Omega}$ definition of eccentricity should give reasonable results when the corresponding orbit is sufficiently Newtonian. The orbits become more relativistic as the total angular momentum associated with the orbit gets smaller. Throughout this paper, we will use the following convention to easily differentiate which EP curves represent large, intermediate and small values of angular momentum. EP curves with dot-dashed lines represent large values of angular momentum, solid lines denote the middle range of angular momentum, and the dashed lines have small values of angular momentum.

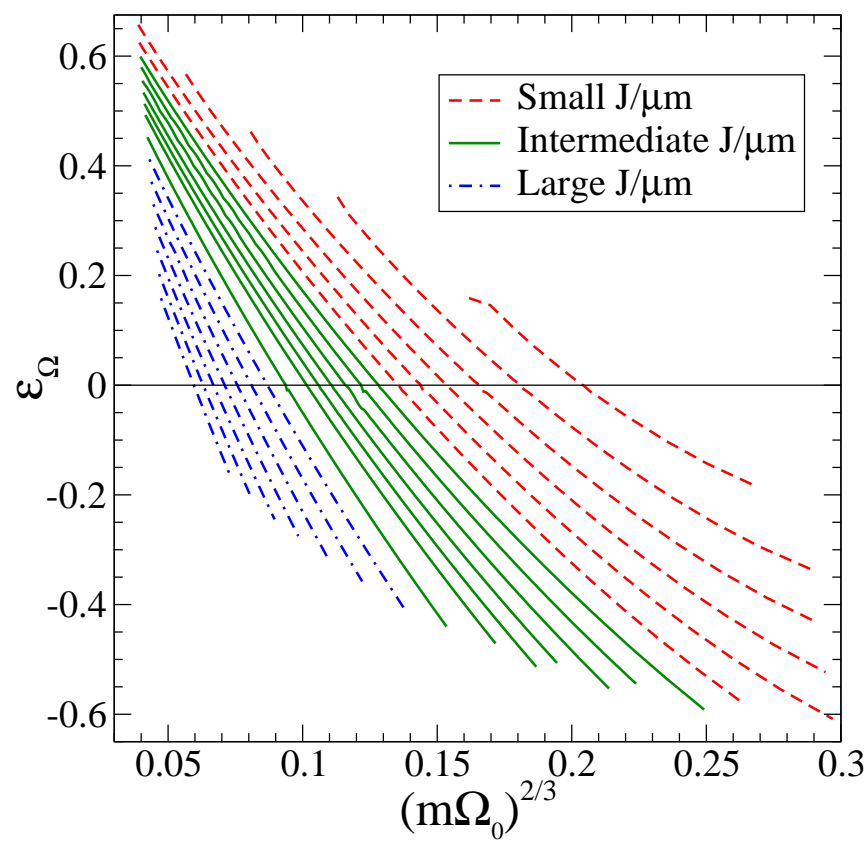

FIG. 3: Eccentricity of non-spinning equal-mass black holes computed along numerical EP curves. The $\epsilon_{\Omega}$ definition of eccentricity is plotted against the orbital angular velocity. Negative values of $\epsilon_{\Omega}$ correspond to models at pericenter, while positive values correspond to apocenter. Large values of the orbital angular momentum are plotted as dot-dashed(blue) lines, intermediate values as solid(green) lines, and small values as dashed(red) lines.

Figure 3 shows only a limited range of eccentricities for each value of angular momentum. There are several reasons for this, both physical and computational. First, to compute the eccentricity, we require data from two corresponding turning points on the same EP curve. That is, we need two points with the same value of the binding energy. Because we do not construct models at arbitrarily large separation, some data at pericenter have no matching data at apocenter. In this case, the eccentricity cannot be computed. Clearly, we cannot compute eccentricities using this method for pericenter data corresponding to unbound orbits, but there there are additional limitations associated with the shape of the effective potential at small separation. Because of strong-field effects, the effective potentials reach a local maximum at small separation (cf. the effective potential for massive test particles orbiting Schwarzschild). For bound orbits, we are limited to computing eccentricities for configurations with energies lower than that of the local maximum for each EP curve. So, the eccentricities plotted in Fig. 3 correspond to data in the neighborhood of the local minimum of each EP curve and extending only as far as the lowest local maximum on either side.

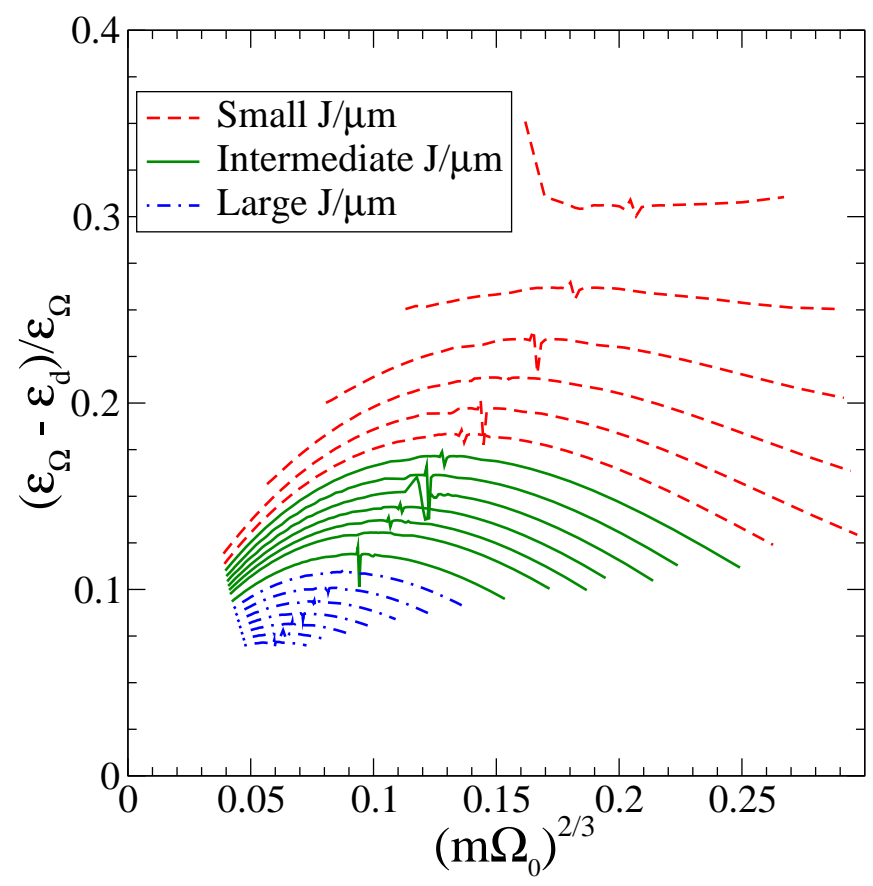

FIG. 4: The relative difference between the $\epsilon_{\Omega}$ and $\epsilon_{d}$ definitions of eccentricity for the non-spinning equal-mass black hole numerical EP curves. Lines as in Fig. 3 .

To test whether the numerical data is behaving as expected, we turn to the definition of eccentricity found in $\epsilon_{d}$. This definition should be reasonable for large separations, but will break down as the coordinate separation of the two black holes decreases because of coordinate effects near the black holes. In Fig. 4, we show the relative difference of the two measurements $\left(\epsilon_{\Omega}-\epsilon_{d}\right) / \epsilon_{\Omega}$. Note that we have manually removed data corresponding to points near the minima of the EP curves since both definitions of eccentricity yield zero at the minimum and the relative error for neighboring points is dominated by numerical noise. However, it is easy to find where those points would have been as the different lines become somewhat jagged in the region of zero eccentricity.

Figure 4 shows the behavior we would have expected. 
For large separations (large $J / \mu m$ ) the coordinate separation $d$ and the orbital angular velocity should both yield reasonable estimates of the eccentricity and we see that the relative error is tending to zero as $J / \mu m$ increases. Clearly, the gauge dependence of the coordinate separation $d$ will cause $\epsilon_{d}$ to become less reliable for smaller separations(small $J / \mu m$ ) and indeed, we see the relative difference increase as $J / \mu m$ decreases. Because the orbital angular velocity $\Omega$ is gauge independent, we expect that $\epsilon_{\Omega}$ will yield a better definition of the eccentricity, however, we need an independent standard against which we can measure the reliability of $\epsilon_{\Omega}$.

\section{B. Post-Newtonian Measurement}

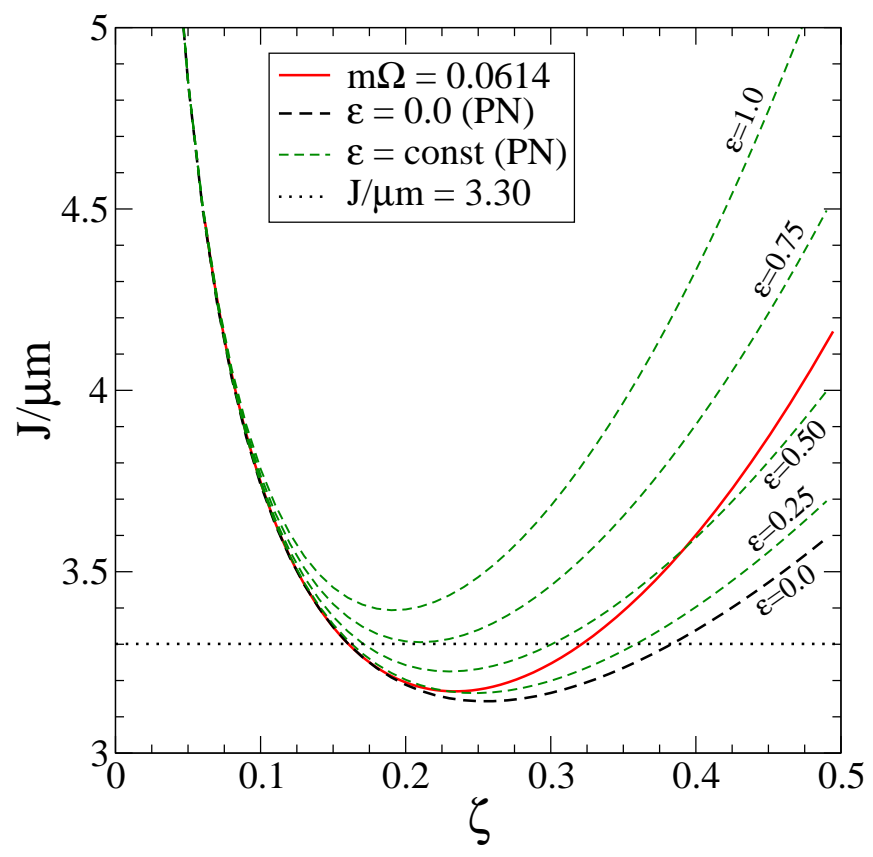

FIG. 5: Parameter space of $J(\epsilon, \zeta)$ (Eq. (10) ) for non-spinning equal-mass black holes. Short-dashed(green or black) lines of constant $\epsilon$ are constructed using the $3 \mathrm{PN}$ equations. The solid(red) line represents all 3PN models with a specific value of $m \Omega=0.0614$ corresponding to a particular numerical model. The horizontal dotted(black) line represents the corresponding orbital angular momentum of that model.

To test $\epsilon_{\Omega}$ in the more relativistic regime we return to post-Newtonian theory. The 3PN equations for the energy and angular momentum in Eqs. (9) and (10) can be used to compute the eccentricity of initial data in several ways, all using information from a single initial-data configuration [12, 23, 24]. The two most useful ways are based on using values for either $E_{b}$ and $\Omega$, or $J$ and $\Omega$ from a given initial-data set. Both methods yield similar but distinct values for the eccentricity. We will denote eccentricities obtained using the energy via $\epsilon_{E_{b}}$, and using the angular momentum via $\epsilon_{J}$.

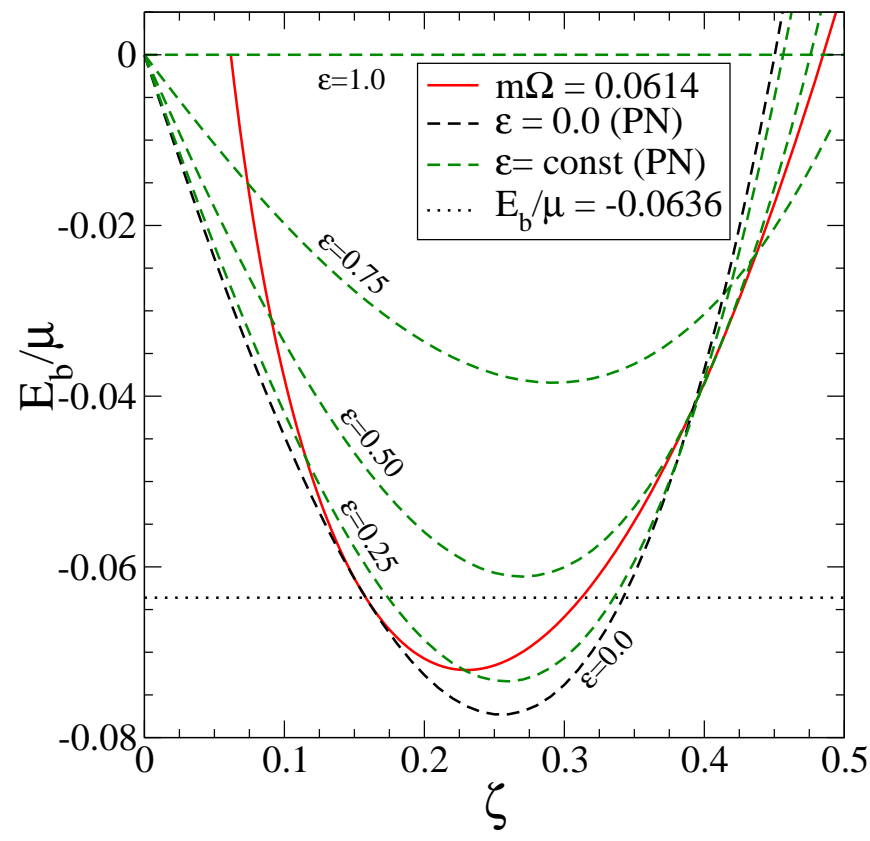

FIG. 6: Parameter space of $E(\epsilon, \zeta)$ (Eq. (9)) for non-spinning equal-mass black holes. Lines as in Fig. 5 except the horizontal dotted(black) line represents the corresponding binding energy of the numerical model.

To use Eqs. (9) and (10) to find eccentricity, we need to simplify the dependencies. Those equations depend on the eccentricity $\epsilon$, the inverse semilatus rectum $\zeta$, and the individual black-hole spins (represented by $\omega$ ). The spin dependence can always be fixed. For now, we consider non-spinning black holes. Next we apply Eq. (14) to replace $\epsilon$ with $m \Omega$ and $\zeta$. The equations now depend on only $m \Omega$ and $\zeta$. To find a $3 \mathrm{PN}$ value of $\zeta$, we set one of the equations (say the energy equation) to the constant (energy) taken from an initial-data set and replace $m \Omega$ with its value from the same data set. We can then use one-dimensional root finding to obtain a value for $\zeta$. Finally, using Eq. (14) again, we can obtain $\epsilon$.

There is an issue when using root-finding methods on the modified equations. Eqs. (9) and (10) are polynomials with multiple roots, so we must determine which value of $\zeta$ to use. Figure 5 shows the parameter space for the 3PN angular momentum from Eq. (10) for the case of non-spinning equal-mass black holes. The angular momenta for lines of constant eccentricity are plotted against $\zeta$ as dashed lines. A solid line shows all 3PN configurations having a constant value of $m \Omega=0.061355$. This value of $m \Omega$ was chosen because it corresponds to the minimum of one of the numerical EP curves. The horizontal dotted line displays the angular momentum from the numerical data set. As can be seen, there are two values of $\zeta$ where these two lines intersect, and both correspond to valid roots of the equation. The smallest positive root corresponds to a very small eccentricity which we would expect for the given data set. The second smallest positive root yields an eccentricity somewhere 
between $\epsilon_{J}=0.25$ and $\epsilon_{J}=0.50$. It is unlikely that the minimum of an EP curve would have such high eccentricities and negative values of $\zeta$ aren't allowed, so we always choose the smallest positive root for $\zeta$. The $3 \mathrm{PN}$ energy from Eq. (9) yields similar results as can be seen in Fig. 6. Again, we always choose the smallest positive root for $\zeta$.

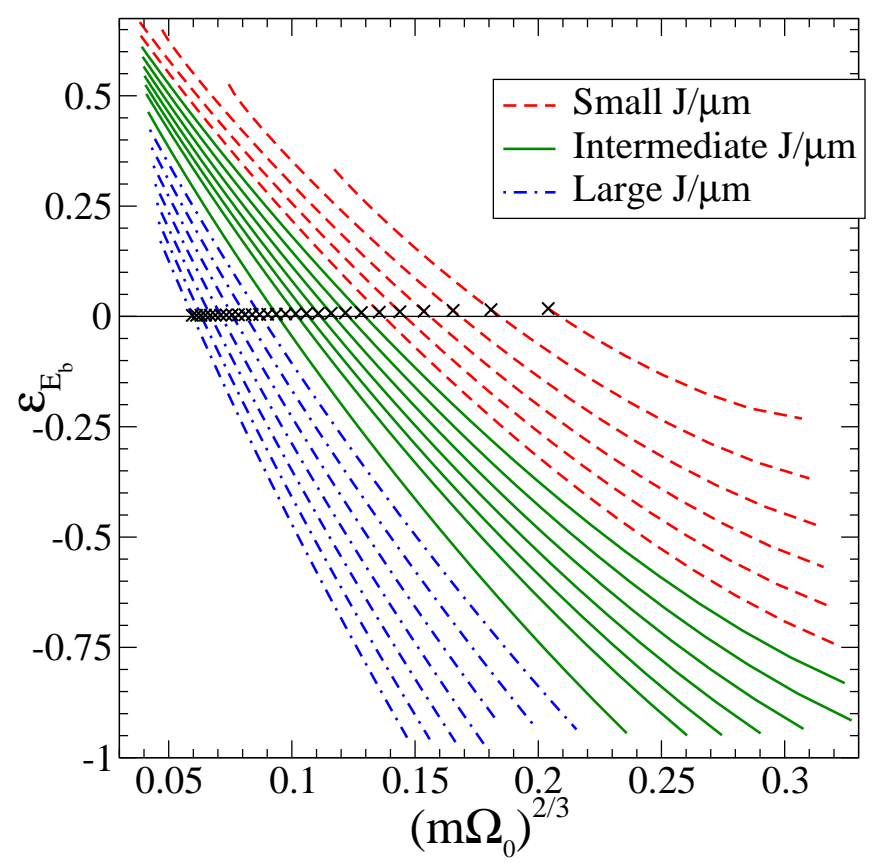

FIG. 7: Eccentricity of non-spinning equal-mass black holes computed along numerical EP curves. The $3 \mathrm{PN} \epsilon_{E_{b}}$ definition of eccentricity is plotted against the orbital angular velocity. Lines are as in Fig. 3. The $\times$ symbols mark the minima of each EP curve.

In Fig. 7 we show the eccentricity $\epsilon_{E_{b}}$ of the same EP curves considered in Fig. 3 but computed using the 3PN energy equation. The minima of the EP curves, which would have $\epsilon_{\Omega}=0$, are marked with $\times$ 's. Qualitatively, these results resemble the previous direct measurements shown if Fig. 3, however there are differences. First, note that the the minima of the EP curves do not correspond exactly to $\epsilon_{E_{b}}=0$ (as first noticed in Refs. [12, 23, 24]). Also, for high values of $J / \mu m, \epsilon_{E_{b}}$ can be evaluated for more of the data points on these EP curves than is possible for $\epsilon_{\Omega}$. The evaluation of $\epsilon_{\Omega}$ was limited in this range because the numerical data did not extend out to sufficiently large separations and $\epsilon_{\Omega}$ requires pairs of turning points to measure the eccentricity. Because $\epsilon_{E_{b}}$ requires information from only a single initial-data set, it can be computed for some data points where $\epsilon_{\Omega}$ cannot. While not presented here, using the angular momentum equation to compute $\epsilon_{J}$ delivers results that are qualitatively similar.

Finally, our goal has been to gauge whether or not $\epsilon_{\Omega}$ was a reasonable definition of eccentricity. In Fig. 8, we show the difference between the $\epsilon_{\Omega}$ and $3 \mathrm{PN}$ definition of eccentricity $\epsilon_{E_{b}}$. We plot $\epsilon_{\Omega}-\epsilon_{E_{b}}$ against $\epsilon_{\Omega}$ rather

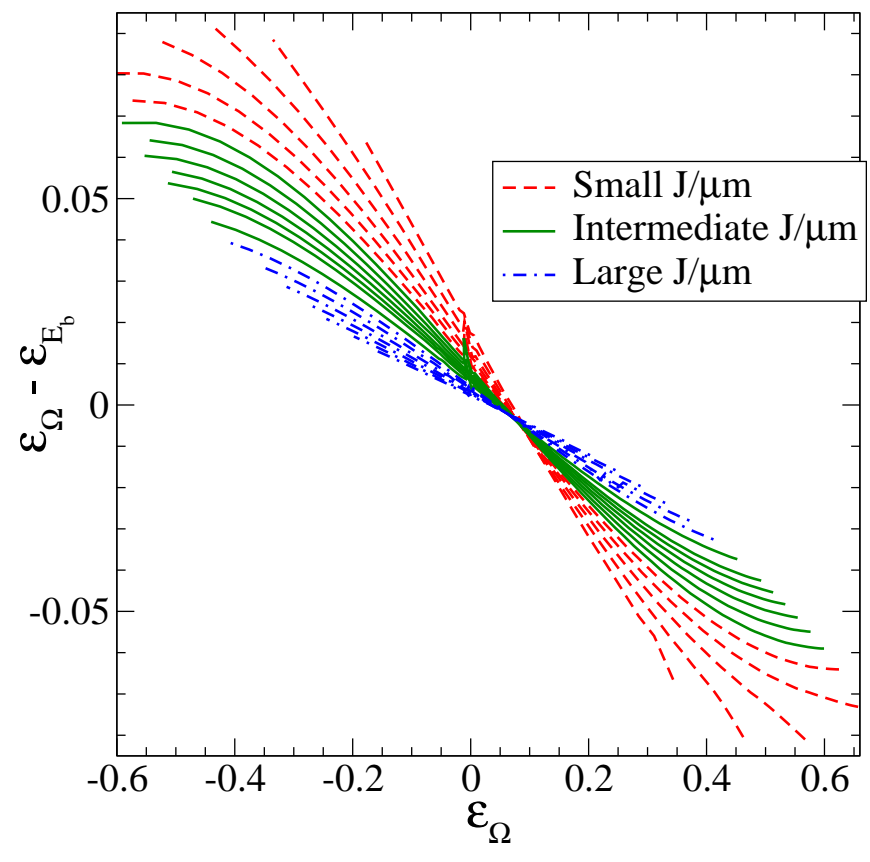

FIG. 8: Difference between $\epsilon_{\Omega}$ and $\epsilon_{E_{b}}$ plotted against $\epsilon_{\Omega}$ for non-spinning equal-mass black holes computed along numerical EP curves. Lines are as in Fig. 3

than a relative difference to avoid division by small number issues that make the graph difficult to read (recall that $\epsilon_{\Omega}$ and $\epsilon_{E_{b}}$ do not evaluate to zero for the same data points). As expected, there is better agreement for more Newtonian configurations (large $J / \mu m$ ) which diminishes as $J / \mu m$ decreases. There is some jaggedness at $\epsilon_{\Omega}=0.0$ caused by the polynomial fitting used to estimate the minima of the EP curves. A careful examination of the apocenter data points (positive $\epsilon_{\Omega}$ ) shows that relative errors for modest values of $\epsilon_{\Omega}$ do not exceed $20 \%$ for even the smallest values of $J / \mu m$. Of course, the relative errors near $\epsilon_{\Omega}=0$ are unbounded. This comparison suggests that the use of either $\epsilon_{\Omega}$ or $\epsilon_{E_{b}}$ yield reasonable measures for the eccentricity when applied to the numerical initial-data sets, although we should be more cautious in trusting results for pericenter data with small values of the angular momentum.

\section{The Komar-Mass Difference}

The first application of the $\epsilon_{E_{b}}$ and $\epsilon_{J}$ definitions to non-spinning, equal-mass black-hole binary initial data was undertaken by Berti et. al.23. In this work, they considered initial data sets that satisfy the Komar-mass criteria for circular orbits and showed that the $3 \mathrm{PN}$ definitions of eccentricity $\epsilon_{E_{b}}$ and $\epsilon_{J}$ yield non-zero results for these configurations which are supposed to be in circular orbits. We reproduce these results in Fig. 9. We note that, while it is true that the circular orbit data have non-vanishing $3 \mathrm{PN}$ eccentricity, the magnitude of 
this eccentricity smoothly approaches zero as the separation increases. We also note, as pointed out in Refs. 12 and [23], that the energy based definition $\epsilon_{E_{b}}$ yields consistently smaller values of eccentricity for the "quasicircular" data than does the angular momentum based definition $\epsilon_{J}$.

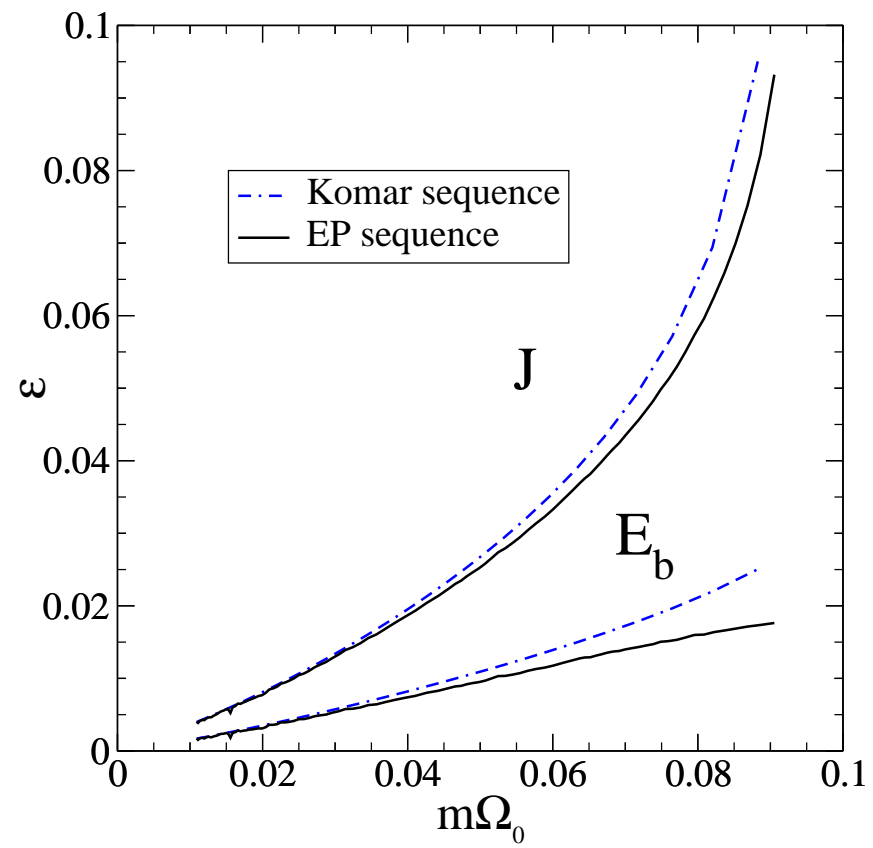

FIG. 9: The 3PN eccentricity measures $\epsilon_{E_{b}}$ and $\epsilon_{J}$ applied to both Komar and EP sequences of non-spinning equal-mass black holes. Note that the EP minima yield quasi-circular data with a smaller eccentricity than is obtained from the Komar-mass ansatz.

In Ref. [10], we showed that circular orbits defined by the EP method yield models that are very similar to those defined by the Komar-mass ansatz. We also showed that, while very similar, all quantitative measurements of the quality of the circular orbits showed that the EP method yields better results. This is again true if we compare the $3 \mathrm{PN}$ eccentricities computed for non-spinning, equal-mass binaries in circular orbits defined by the EP method and the Komar-mass ansatz. The results are shown in Fig. 9, where it is clear that the the EP method yields consistently smaller values of eccentricity for quasi-circular orbit data.

Although the EP method yields consistently better results for circular orbits than can be obtained using the Komar-mass method, the differences are in general not sufficiently significant to outweigh the considerable computational expense associated with the EP method. When we compute eccentricities using the $\epsilon_{\Omega}$ definition, the overhead of using the EP method is even larger. It would be useful to find another means of estimating the eccentricity of binary initial data.

In Fig. 10, we again plot the eccentricity $\epsilon_{\Omega}$ of the same set of non-spinning, equal-mass initial data. However, on the horizontal axis, we plot the dimensionless Komar-

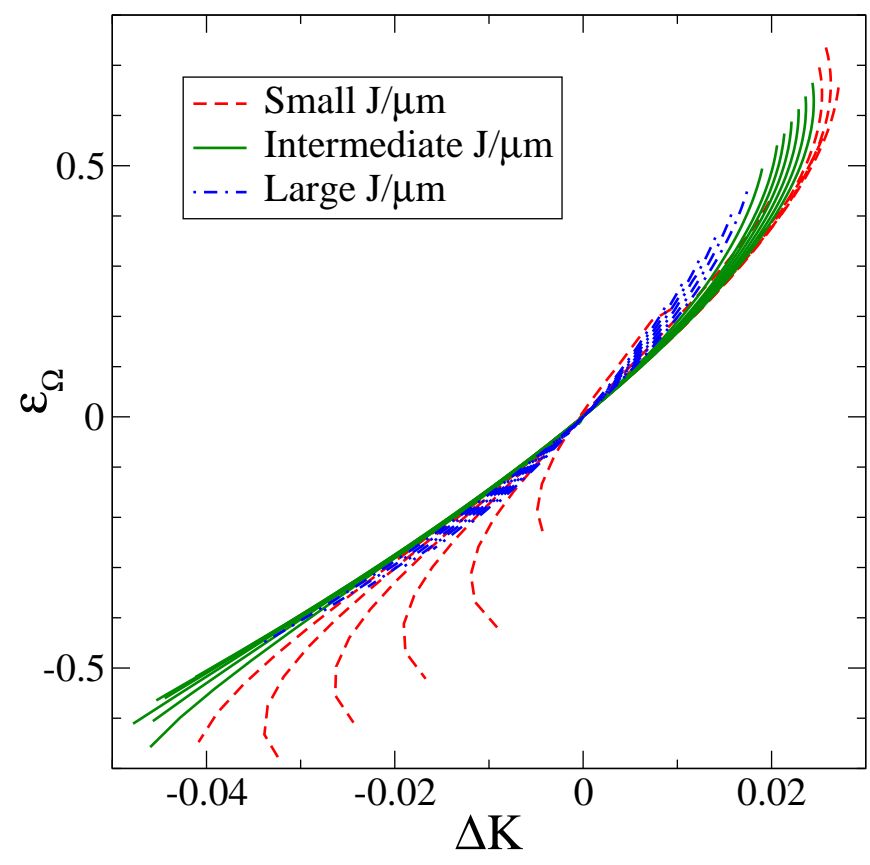

FIG. 10: The eccentricity measure $\epsilon_{\Omega}$ applied to non-spinning equal-mass black holes computed along numerical EP curves and plotted against the Komar-mass difference $\Delta K$. Lines are as in Fig. 3 .

mass difference

$$
\Delta K \equiv\left(E_{A D M}-M_{K}\right) / \mu .
$$

We see a very strong correlation in the data, though the correlation weakens as we move further from quasicircular orbits. It is worth noting that the EP curves with low $J / \mu m$ curve back towards zero $\Delta K$ for configurations with negative eccentricities (pericenter) and small $\mathrm{J} / \mu \mathrm{m}$. This behavior is for data in the region of the local maxima in the EP curves. That this occurs is consistent with the notion that these local maxima represent unstable quasi-circular orbits, and hence we will find the Komar-mass difference vanishing in this region. More importantly, it shows that all of the definitions of eccentricity we have used will break down in this highly relativistic region.

\section{COROTATION}

So far we have restricted ourselves to the case of nonspinning black holes. However, corotating configurations have received considerable attention in spite of the fact that we do not expect to see corotating black holes in nature. In addition to the non-spinning case, Berti et. al. [23] also computed the $3 \mathrm{PN}$ eccentricities for the corotating data presented in Ref. [10] and we reproduce these results in Fig. 11.

One of the primary reasons that the initial data for corotating black holes has been studied so extensively 
is that there is a simple and unique means of enforcing the condition of corotation on the black holes. This is in contrast to any attempt to enforce a specific value of spin (even no spin) on each black hole, which necessarily includes the uncertainty in how we define the spin of an individual black hole in a binary configuration.

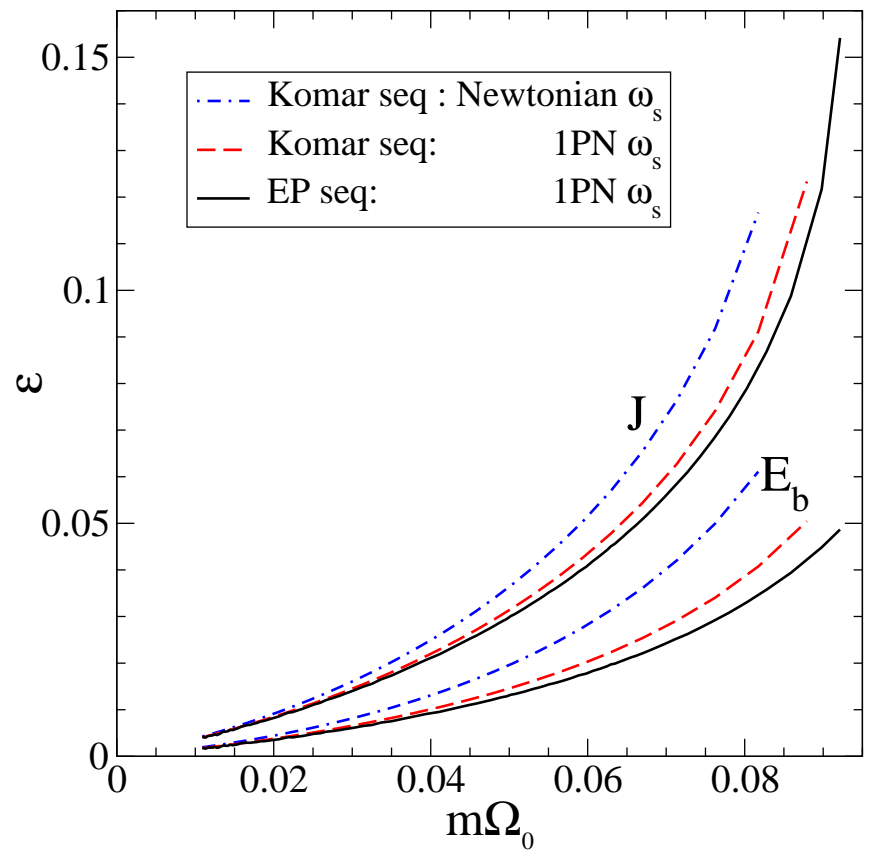

FIG. 11: The 3PN eccentricity measures $\epsilon_{E_{b}}$ and $\epsilon_{J}$ applied to both Komar and EP sequences of corotating equal-mass black holes. The dot-dashed(blue) lines correspond to quasicircular data defined via the Komar-mass ansatz and where the Newtonian notion of corotation is used in the 3PN equations. The dashed(red) lines show the improvement obtained by including the $1 \mathrm{PN}$ correction to the notion of corotation. The solid(black) lines show the added improvement of using quasi-circular data based on the EP method.

The Newtonian concept of corotation implies that each black hole rotates with a spin angular velocity $\omega_{s}$ that is equal to the orbital angular velocity. This Newtonian notion of corotation (i.e. $\omega_{s}=\Omega_{0}$ ) was used to fix the spin parameters in Eqs. (9) and (10) when using these $3 \mathrm{PN}$ equations to compute $\epsilon_{E_{b}}$ and $\epsilon_{J}$. To our knowledge, this Newtonian notion of corotation has been used in all PN computations dealing with corotation (cf Refs. [12, 23, 25, 26, 27, 28]). However, in Ref. [10], we have shown that there are relativistic corrections to the spin angular velocity associated with corotating black holes. We find $\omega_{s}$, including the $1 \mathrm{PN}$ correction, to be of the form

$$
\omega_{s}=\Omega_{0}\left(1-\eta\left(m \Omega_{0}\right)^{2 / 3}+\cdots\right),
$$

where $\eta=\mu / m$ is the symmetric mass ratio which takes the value of $1 / 4$ for equal-mass binaries. Also shown in Fig. 11 are the two 3PN eccentricities computed using the corrected definition for $\omega_{s}$. We find that using this corrected definition significantly decreases the 3PN estimated eccentricity for these circular-orbit models.
As with the non-spinning case, we can also compute the eccentricities for the corotating equal-mass binaries in quasi-circular orbits defined in terms of the EP method rather than the Komar-mass ansatz. Including also the improved definition for $\omega_{s}$ in the $3 \mathrm{PN}$ definitions of eccentricity, we find the evaluated eccentricities are smallest when evaluated for quasi-circular data defined by the EP method. This can also be seen in Fig. 11

Even with the correction to $\omega_{s}$, we notice the magnitude of the $3 \mathrm{PN}$ eccentricities computed for "circular" data are consistently larger for corotating binary data than for non-spinning data. We cannot be certain why this is the case, but we should keep in mind that there is an inherent inconsistency in any attempt to attach the notion of eccentricity to corotating configurations. All of our definitions for eccentricity, including the $3 \mathrm{PN}$ definitions that can be evaluated using information from a single data set, ultimately rely on information from both a pericenter and an apocenter configuration. For eccentric orbits, the spins of corotating black holes will change from pericenter to apocenter. Since the spins (including spin-orbit and spin-spin couplings) contribute to the total energy, this variation of the spin throughout the orbit must impact upon our definitions of eccentricity. This is likely to cause few problems when considering nearly circular orbits (where the spin varies little from pericenter to apocenter), but our definitions of eccentricity may not yield reasonable results for orbits that deviate significantly from being circular.

\section{CONCLUSIONS}

In this paper, we have examined several basic questions associated with the construction of binary blackhole initial data. When we construct binary initial-data sets using the extended CTS equations and fix the freelyspecifiable parts of the data and boundary conditions to be consistent with the assumptions of quasiequilibrium, we obtain models for black holes in circular orbits. But what happens if we set aside the quasiequilibrium assumption that imposes circular orbits? The resulting data can no longer evolve in a quasiequilibrium manor as the orbit will have a significant eccentricity. Our investigations suggest that the initial data models we obtain represent, in general, binaries that are at turning points (either apocenter or pericenter) of some general eccentric orbit.

The specific notion of quasiequilibrium that we set aside is implemented by imposing either the Komar-mass condition or by choosing the minimum of an EP curve as our circular orbit model. If, as it seems, general initialdata models on an EP curve are at turning points, then we can use information from these models to estimate the eccentricity of the model's orbits. Of course, there is no unique definition of eccentricity. We have examined several possible definitions for eccentricity. Using only information from the initial-data sets on an EP curve, 
we have defined two eccentricities for an orbit $\left(\epsilon_{\Omega}\right.$ and $\epsilon_{d}$ ), but these definitions require that we have representative models at both the pericenter and apocenter turning points of a given orbit (assumed to have constant binding energy). We have compared these definitions of eccentricity to the $3 \mathrm{PN}$ definitions $\left(\epsilon_{E_{b}}\right.$ and $\left.\epsilon_{J}\right)$ developed by Mora and Will 12$]$. All of the definitions agree quite well for non-relativistic orbits. They are also in reasonably good agreement for more tightly-bound and relativistic orbits as well, although the results are quantitatively different.

One might ask which definition is better for more relativistic situations. However, it isn't clear that there is a meaningful answer. In comparing the numerical and 3PN eccentricity measures for relativistic cases, we have noticed an interesting and unexpected feature of the $3 \mathrm{PN}$ equations. Figure 2 compares numerical and 3PN EP curves for equal-mass non-spinning black-hole binaries. The EP curves each have a constant value for the orbital angular momentum. All of the numerical EP curves cover a finite range of separations (parameterized by $m \Omega_{0}$ ). This is because it becomes computationally expensive to compute models at very large separations (small $m \Omega_{0}$ ) and it becomes increasingly difficult to obtain convergent solutions at very small separations. What was unexpected is that the $3 P N$ EP curves also cover a finite range of separations.

For sufficiently small values of $J / \mu m$, the $3 \mathrm{PN}$ EP curves do not extend to configurations with arbitrarily large separation. To make this clear, Fig. 2 includes a curve that marks the boundary (for both large and small separation) of the $3 \mathrm{PN}$ EP curves. This is most easily seen for the 3PN EP curves near the bottom of Fig. 2 , Here we can see that the 3PN EP curves do not extend to arbitrarily small values of $m \Omega_{0}$, but the numerical EP curves do (although we do not compute them for arbitrarily large separation).

That this behavior is not an artifact of our method for computing the 3PN EP curves can be seen by examining Fig. 5. Recall that angular momentum is held constant along EP curves, so an EP curve is represented by a horizontal line in this figure. Notice that all curves of constant eccentricity have a local minimum. For $J / \mu m \gtrsim 3.4$, an EP curve can extend from $\epsilon=0 \rightarrow 1$. However, for $J / \mu m \lesssim 3.4$, can only extend from $\epsilon=0$ to a maximum eccentricity that is less than 1 , and which is determined by which curve of constant $\epsilon$ has its minimum tangent to the EP curve.

It is physically reasonable that large eccentricity orbits should not exist as the orbits become sufficiently relativistic since the finite size of the black holes would lead to a collision. However, the PN equations treat the black holes as point particles and should not be sensitive to this limitation. Also, we remind the reader that the numerical EP curves have no difficulty in extending to large separation $(\epsilon \rightarrow 1)$ for relativistic orbits. This suggests that, for relativistic orbits, there may be a problem with the definition of eccentricity and semilatus rectum used by Mora and Will 12] to parameterize the PN energy and angular momentum. We do not consider this to be a serious problem, but rather an indication that any definition of eccentricity is of limited value for orbits where the interaction between the black holes becomes highly relativistic.

Given the strong correlation between the various measures of eccentricity and the difference between the Komar mass and the ADM energy as measured by $\Delta K$ and seen in Fig. 10, we suggest that perhaps $\Delta K$ can serve as a useful invariant means of parameterizing the eccentricity of an orbit. However, initial-data studies can at best suggest possible useful parameterizations. It will be most useful to evolve initial data that are significantly eccentric and examine the orbital dynamics to better understand both the parameterization of eccentricity and its effects on the dynamics. For example, it may be particularly interesting to explore the evolution of eccentric initial data for an "orbit" that has no pericenter turning point on its EP curve.

\section{Acknowledgments}

We would like to thank Harald Pfeiffer, Alessandra Buonanno, Clifford Will, and Emanuel Berti for useful discussions. This work was supported by NSF Grant No. PHY-0555617 to Wake Forest University. G.C. acknowledges support from the Z. Smith Reynolds Foundation. Computations were performed on the Wake Forest University DEAC Cluster with supported from an IBM SUR grant and the Wake Forest University IS Department.
[1] F. Pretorius, Phys. Rev. Lett. 95, 121101 (2005).

[2] M. Campanelli, C. O. Lousto, P. Marronetti, and Y. Zlochower, Phys. Rev. Lett. 96, 111101 (2006).

[3] J. G. Baker, J. Centrella, D. Il Choi, M. Koppitz, and J. van Meter, Phys. Rev. Lett. 96, 111102 (2006).

[4] P. Diener, F. Herrmann, D. Pollney, E. Schnetter, E. Seidel, R. Takahashi, J. Thornburg, and J. Ventrella, Phys. Rev. Lett. 96, 121101 (2006).

[5] P. C. Peters, Phys. Rev. 136, B1224 (1964).

[6] G. B. Cook, Phys. Rev. D 50, 5025 (1994).
[7] E. Gourgoulhon, P. Grandclément, and S. Bonazzola, Phys. Rev. D 65, 044020 (2002).

[8] R. Arnowitt, S. Deser, and C. W. Misner, in Gravitation: An Introduction to Current Research, edited by L. Witten (Wiley, New York, 1962), pp. 227-265.

[9] A. Komar, Phys. Rev. 113, 934 (1959).

[10] M. Caudill, G. B. Cook, J. D. Grigsby, and H. P. Pfeiffer, Phys. Rev. D 74, 064011 (2006).

[11] M. L. Skoge and T. W. Baumgarte, Phys. Rev. D 66, 107501 (2002). 
[12] T. Mora and C. M. Will, Phys. Rev. D 69, 104021 (2004).

[13] G. B. Cook and H. P. Pfeiffer, Phys. Rev. D 70, 104016 (2004).

[14] J. W. York, Jr., Phys. Rev. Lett. 82, 1350 (1999).

[15] H. P. Pfeiffer and J. W. York, Jr., Phys. Rev. D 67, 044022 (2003).

[16] G. B. Cook, Phys. Rev. D 65, 084003 (2002).

[17] A. Ashtekar and B. Krishnan, Phys. Rev. D 68, 104030 (2003).

[18] A. Ashtekar, C. Beetle, O. Dreyer, S. Fairhurst, B. Krishnan, J. Lewandowski, and J. Wiśniewski, Phys. Rev. Lett. 85, 3564 (2000).

[19] J. L. Jaramillo, E. Gourgoulhon, and G. A. M. Marugán, Phys. Rev. D 70, 124036 (2004).

[20] T. W. Baumgarte, Phys. Rev. D 62, 024018 (2000).

[21] H. P. Pfeiffer, S. A. Teukolsky, and G. B. Cook, Phys.
Rev. D 62, 104018 (2000).

[22] D. Christodoulou, Phys. Rev. Lett. 25, 1596 (1970).

[23] E. Berte, S. Iyer, and C. M. Will, Phys. Rev. D 74, 061503(R) (2006).

[24] T. Mora and C. M. Will, Phys. Rev. D 66, 101501(R) (2002).

[25] T. Damour, E. Gourgoulhon, and P. Grandclément, Phys. Rev. D 66, 024007 (2002).

[26] L. Blanchet, Phys. Rev. D 65, 124009 (2002).

[27] M. Campanelli, C. O. Lousto, and Y. Zlochower, Phys. Rev. D 74, 084023 (2006).

[28] L. Blanchet, in 2001: A Relativistic Spacetime Odyssey, edited by I. Ciufolini, D. Dominici, and L. Lusanna (World Scientific, River Edge, U.S.A., 2003), p. 411. 